\title{
CARACTERIZACION AMBIENTAL Y PRODUCTIVA DE RODALES FORESTALES DE CASTAÑO EN CHILE
}

Susana Benedetti, Jaime Saavedra'

\section{RESUMEN}

El interés de pequeños y medianos propietarios por opciones forestales rentables, que no requieran grandes superficies y que ofrezcan diversos productos, junto a los antecedentes del potencial productivo y económico de Castaño (Castanea sativa) en Chile y el fomento a la diversificación forestal, posicionan a la especie como una alternativa real de inversión.

Castaño puede crecer en distintas zonas entre las regiones VII del Maule a X de Los Lagos, en sitios que presenten condiciones de suelo y clima aptos a la especie. Las regiones $X$ de los Lagos y IX de La Araucania son las que presenta las mejores condiciones ambientales para el desarrollo de plantaciones forestales de castaño y, si bien existen plantaciones desde la VII a la X Regiones, es la Región de Los Lagos, la que concentra la mayor cantidad de plantaciones y de mayores edades, entre 15 a 25 años. El análisis de estas plantaciones generará los antecedentes que permitirán orientar decisiones de plantación y manejo con mayor precisión. Este estudio presenta el análisis y caracterización realizado en siete rodales de castaño de edades entre 10 y 28 años, ubicados entre la VII Región del Maule a la X Región de Los Lagos, sobre características ambientales, dasométricas, de forma y sanidad de asociación vegetacional.

Palabras clave: Castanea sativa, variables ambientales, variables dendrométricas.

\footnotetext{
Susana Benedetti (sbenedet@infor.cl), Instituto de Investigación Forestal. INFOR. Huérfanos 554, Santiago, Chile. Jaime Saavedra (jsingfor@yahoo.com), consultor privado.
} 


\section{SUMMARY}

Small and medium landowners in Chile are interested in profitable forest activities that do not require large extend of land and at the same time would be able to offer different products. Besides that, according to the references, Chestnut tree (Castanea sativa) growing in Chile has an wide productive and economic potential, and also the species is included in the National Forest Diversification Program, becoming a real investment option.

Chestnut tree may grow at different zones from Maule ( $7^{\mathrm{a}}$ administrative region) to Los Lagos $\left(10^{\mathrm{a}}\right.$ administrative region) where site conditions (soil and climate) allow it. Administrative regions $9^{\mathrm{a}}$ and $10^{\mathrm{a}}$ present the best growing conditions to establishment of forest plantation, even though plantations are distributed from VII to X Regions. The X Region concentrates the larger amount of forest plantations and also the older ones ( 15 to 40 years old). The analysis of these plantations is the base for orienting decisions of plantation and forest management more accurately. This study presents the results of analyzing and characterizing seven chestnut plantations located between VII to X Regions with ages from 10 to 28 years old. The variables evaluated are environmental characteristics, biometric, shape, sanitary status and vegetational association.

Key words: Castanea sativa, environmental variables, tree variables 


\section{INTRODUCCION}

El atractivo de castaño como opción forestal para pequeños y medianos propietarios radica en su caracteristica de especie multipropósito, productora de madera de alto valor y de frutos, estos últimos son anuales y representanuna via de ingresos periódicos en espera del ciclo de cosecha de la madera. Otra caracteristica de interés para los propietarios es la capacidad de rebrote y el vigor del castaño, lo que permite un manejo sostenido en el tiempo.

Los antecedentes sobre la adaptación y desarrollo de la especie en Chile, permitieron además la incorporación de castaño en la política de fomento forestal, un elemento importante para cualquier propietario al momento de decidir la inversión en plantaciones forestales.

En este contexto el presente trabajo analiza las condiciones de sitio, de crecimiento y de sanidad de siete rodales de castaño existentes en el área definida como potencial para el desarrollo de plantaciones forestales de la especie en Chile, distribuidos entre la VII y X Regiones, abarcando una amplia área geográfica y por tanto una variedad de condiciones ambientales.

\section{OBJETIVOS}

Caracterizar las estaciones ecológicas y la adaptación y crecimiento de rodales de castaño, distribuidos en la zona potencial determinada para plantaciones de la especie en Chile, a fin de precisar las condiciones ambientales y de desarrollo que permitan orientar el manejo orientado ala producción de madera de alto valor.

\section{ANTECEDENTES GENERALES}

Castaño, de nombre cientifico Castanea sativa Miller, pertenece a la familia Fagaceae y al género Castanea. Este género está compuesto por 13 especies originarias de las regiones templadas del hemisferio norte, entre las cuales Castanea sativa es la única presente en Europa, distribuida en Turquia, Grecia, Italia, Francia, España y Portugal. Cinco son originarias del Asia oriental (C. mollisima, C. crenata, C. henryi, $C$ segunii y $C$. davidii) y siete de norteamérica ( $C$. dentata, $C$. ozarkensis, $C$. ashei, C. paucispina, C. pumila, C. floridiana y C. alnifolia) (Berrocal, et al., 1998).

Castaño (Castanea sativa) es un árbol caduco y longevo, capaz de alcanzar entre 20 y 30 metros de altura. Su sistema radicular es profundizador, robusto y extendido, lo que favorece el suministro de agua y nutrientes al árbol. Posee alta capacidad para rebrotar, tanto de tocón como de raiz, por lo que tiende a formar masas puras (Berrocal et al, 1998).

En cuanto a sus requerimientos ecológicos, necesita un mínimo de cinco meses de temperatura media superior a los $10^{\circ} \mathrm{C}$ (Medel, 1986) y temperaturas medias anuales de $8^{\circ}$ a $15^{\circ} \mathrm{C}$ (Bagnaresi, 1986; Sudzuki, 1983 cit. por Loewe et al. 1994). Requiere de una precipitación media anual minima de $700 \mathrm{~mm}$. (Bagnaresi, 1986; Bourgeois, 1992), suelos profundos (Bourgeois, 1992), desde $60 \mathrm{a} 150 \mathrm{~cm}$, texturas medias a livianas, buen drenaje y no son recomendables $\mathrm{pH}$ superiores a 6,5 , debido a que ocasionan problemas de clorosis (Bourgeois, 1992). Crece bien en un amplio espectro de altitud, desde 0 a $1000 \mathrm{msnm}$. 
El crecimiento del Castaño en las plantaciones forestales existentes en Chile logra incrementos diamétricos mayores a $1 \mathrm{~cm}$ por año. Los individuos presentan excelente forma y vigor, clara dominancia apical, buena poda natural y buenas condiciones sanitarias. La superficie potencial de cultivo entre la VII y X Regiones supera los 2 millones de hectáreas cuando crece sin riego y es mayor a los 3 millones cuando se cultiva en suelos regables (Benedetti y Subiri, 2000). A estas ventajas comparativas respecto a muchas especies que crecen en Chile se agrega que a nivel mundial su madera es reconocida por su versatilidad, color claro y uniforme y su marcada veta, lo que le confiere un aspecto superficial muy agradable. Presenta alta durabilidad natural y buena resistencia mecánica, con características muy parecidas a las del lingue (Persea lingue) del bosque nativo chileno, por lo que se puede utilizar para carpinteria y fabricación de muebles, puertas, ventanas, escaleras, pisos o parquet, chapas y revestimientos interiores. Todo lo anterior se debe a la buena trabajabilidad de su madera, se la puede tornear, cilindrar, taladrar, cepillar y lijar sin problemas. En aserrio alcanza rendimientos volumétricos de 50 a $60 \%$ y se deja secar bien, especialmente si previo al secado artificial se le somete a un secado natural hasta un contenido de humedad cercano al 50\% (Zanuttini y Cielo, 1996; Cabrera, 1998; Juacida et al, 1999).

Desde el punto de vista de plagas y enfermedades la especie es susceptible al ataque de diversos patógenos entre los que destacan la enfermedad de la tinta (Phytophtora cinnamoni) y el cancro o cáncer bacteriano (Endothia parasitica), responsables este último de ocasionar fuertes ataques a los castañares de Europa y Norteamérica. En Chile no está presente este último patógeno y si bien hay presencia de Phytophtora, no se ha apreciado a la fecha un daño importante causado por este hongo.

Otro defecto común en bosques de Castaño en Europa, que tampoco se ha apreciado en Chile es la acebolladura de la madera. Esta se manifiesta como un agrietamiento entre anillos de crecimiento ya sea en una parte del perímetro del fuste o, en el caso más grave, en la circunferencia completa, lo que genera la separación del tronco (Macchioni y Pividori, 1996; Amorini et al, 1997).

Lo que si ataca a Castaño en Chile, al igual que a otras especies como Eucalytus nitens , E. globulus, algunas especies nativas y también a huertos frutales, es la chicharra (Tettigades chilensis). En este caso, las plantaciones juveniles no lignificadas son altamente vulnerables ya que la hembra pone los huevos en los de tejidos blandos y suculentos, provocando daños por deformaciones y, en algunos casos, pérdidas por mortalidad. Sin embargo, en general, los daños provocados no son significativos desde el punto de vista productivo (Parra y González, 1998).

En relación al mercado de la madera, el mercado de Europa es el más desarrollado. El mercado nacional se caracteriza por la estrechez e irregularidad de la oferta, demanda insatisfecha y la aceptación del producto por parte de la industria transformadora y consumidores finales.

Los principales productores de madera de Castaño se encuentran en Francia, Italia y Grecia. Francia es el principal proveedor de revestimientos de madera, parquet y contrachapados de Castaño para Bélgica, Holanda y Alemania. Del mismo modo, es el 
principal proveedor de madera aserrada y en trozos para Portugal (50.000 t/año), Italia (43.000 t/año) y España (15.000 t/año). Italia es el segundo productor de madera de Castaño de la Comunidad Económica Europea, con una cosecha anual aproximada de $900.000 \mathrm{~m}^{3}$. Además de importar madera, exporta tableros tipo placa carpintera, puertas de cocina y amoblados en general.

En cuanto a precios, en Francia la materia prima para el foliado ( $2 \mathrm{~m}$ de largo, 35 $40 \mathrm{~cm}$ de diametro) se transa a valores entre 274 y 438 US $\$ / \mathrm{m}^{3}$; trozas para muebles con diámetros mayores a $30 \mathrm{~cm}$, alcanzan los US $\$ 110 / \mathrm{m}^{3}$ a orilla de camino; con diámetro menor a $18 \mathrm{~cm}$ y largo entre 2,2 y $3,0 \mathrm{~m}$, US $\$ 70 / \mathrm{m}^{3}$. Por último, trozas de $10 \mathrm{~cm}$ de diámetro y $1,1 \mathrm{~m}$ de largo, utilizados para la confección de parquet, alcanzan precios de US $\$ 24 / \mathrm{m}^{3}$ (Centre Regional de la Propiete Forestiere, 1995, citado por Cabrera, 1998). En Chile, el Castaño es utilizado para aserrio y su uso final es la industria del mueble, siendo sus precios comparativamente altos en relación con otras maderas que se comercializan en el país (Benedetti y Saavedra, 2003).

Finalmente, el cultivo forestal de la especie en Chile permite obtener a la cosecha importantes ingresos por su madera, que se destina a abastecer un mercado en actividad a pesar de las restricciones impuestas por la heterogeneidad

\section{MATERIAL Y METODO}

Se seleccionó 7 rodales de castaño en el área en que existen en Chile plantaciones con objetivos de producción forestal. Cada rodal se caracterizó en base a las siguientes variables:

- Variables Ubicación: altitud, coordenadas geográficas, exposición, pendiente.

- Variables climáticas: distrito agroclimático, temperatura media anual, temperatura media máxima, temperatura media mínima, precipitación media anual, heladas, meses no húmedos $\mathrm{y} / \mathrm{o} \mathrm{secos}$.

- Variables edáficas: profundidad, pedregosidad, textura, horizonte orgánico, densidad aparente, estructura, macro y microelementos, $\mathrm{pH}$, capacidad de intercambio cationico.

- Variables dasométricas, de sanidad y de forma: Diámetro a la altura del pecho (DAP), dominancia, altura de individuos dominantes y codominantes, cobertura de copa, sanidad, rectitud, presencia de flechas.

Para rectitud se definió tres criterios: 1: bueno; 2 : regular; 3 : malo

Para dominancia se definió cuatro criterios: 1 : dominante; 2 : codominante; 3 : intermedio y 4 : suprimido.

Para flecha se utilizó dos criterios: 1: presenta dos o más flechas; 0 : no presenta flechas. Para la variable sanidad se consideró daño mecánico, presencia de hongos, daño de insectos y tres criterios: 1 : sano; 2: regular; 3 : malo

Para cobertura de copa se empleó 4 rangos: $0-25 \% ; 25-50 \% ; 50-75 ; 75-100 \%$ 
Conjuntamente a lo anterior se describió la vegetación acompañante identificando las principales especies. La identificación de cada rodal se presenta en el Cuadro $\mathrm{N}^{\circ} 1$.

\section{Cuadro $\mathrm{N}^{\circ} 1$}

IDENTIFICACIÓN DE RODALES DE CASTAÑO ANALIZADOS

\begin{tabular}{|l|l|l|l|l|}
\hline Rodal & Región & Provincia & Zona & Año de plantación \\
\hline Alupenhue & VII & Cunco & Precordillera & 1998 \\
\hline Lanalhue & VIII & Arauco & Costa & 1979 \\
\hline Santa Luisa & $\mathrm{IX}$ & Malleco & Precordillera & 1980 \\
\hline Las Minas & $\mathrm{X}$ & Valdivia & Costa & 1978 \\
\hline Pillo Pillo & $\mathrm{X}$ & Valdivia & Costa & 1980 \\
\hline Pumillahue & $\mathrm{X}$ & Valdivia & Valle Central & 1982 \\
\hline San Pedro & $\mathrm{X}$ & Valdivia & Valle Central & 1980 \\
\hline
\end{tabular}

Para la definición de las variables de ubicación se visitó cada uno de los rodales y se midió en terreno con GPS las coordenadas geográficas, la altitud y la exposición, para la medición de la pendiente se utilizó un clinómetro.

Para las variables edáficas se realizó una calicata en cada lugar, en terreno se midió profundidad, pedregosidad, altura de litera, profundidad del horizonte orgánico. Para el análisis físico se extrajo una muestra de la calicata, un pan de $40^{\star} 40^{\star} 40 \mathrm{~cm}$. para la definición de la estructura del suelo, para la densidad aparente se extrajó tres muestras de los primeros $30 \mathrm{~cm}$ del perfil de la calicata. El análisis químico se realizó en muestras tomadas con barreno a $60 \mathrm{~cm}$ de profundidad en tres puntos del rodal.

En el caso de las variables dasométricas y de forma, en cada rodal se definió una parcela circular de $200 \mathrm{~m}^{2}$, a todos los individuos de la parcela se les midió directamente en terreno DAP, rectitud y flecha, a cada individuo además se le evaluó la sanidad. Para la altura se consideró 6 árboles de los estratos dominantes y codominantes a los cuales se les midió altura total con un hipsómetro.

Posteriormente esta información se traspasó a una ficha por rodal acompañada por el análisis de suelo correspondiente, con las cuales se realizó el análisis de resultados.

\section{RESULTADOS Y DISCUSION}

Los rodales de castaño estudiados, como muestra el Cuadro $\mathrm{N}^{\circ} 2$, se localizan en altitudes entre los 44 y 721 msnm y entre 0 y $18 \%$ de pendiente. En cuanto a exposición se presentan en distintas situaciones, no siendo esta variable una limitante para su establecimiento. Estas caracteristicas son concordantes con lo que señala la bibliografía respecto a la especie. 


\section{Cuadro $\mathrm{N}^{\circ} 2$}

\section{VARIABLES DE UBICACION DE LOS RODALES DE CASTAÑO ANALIZADOS}

\begin{tabular}{|c|c|c|c|c|c|c|}
\hline Rodal & Region & $\begin{array}{l}\text { Coordenada } \\
\text { Geográfica }\end{array}$ & $\begin{array}{l}\text { Año de } \\
\text { plantación }\end{array}$ & $\begin{array}{l}\text { Altitud } \\
\text { (msnm) }\end{array}$ & Exposicion & $\begin{array}{l}\text { Pendiente } \\
(\%)\end{array}$ \\
\hline Alupenhue & VII & $\begin{array}{l}35^{\circ} 14^{\prime} 43,77^{\circ} \mathrm{S}, 71^{\circ} \\
5^{\prime} 7,69^{\prime} \mathrm{O}\end{array}$ & 1998 & 554 & Extendida, Sur & $0-5$ \\
\hline Lanalhue & VIII & $\begin{array}{l}37^{\circ} 54^{\prime} 52,45^{\circ} \mathrm{S}: 73^{\circ} \\
222^{\prime} 6,38^{\circ} \mathrm{O}\end{array}$ & 1979 & 260 & Sur & 18 \\
\hline Santa Luisa & IX & $\begin{array}{l}38^{\circ} 4^{\prime} 9.93^{\circ} \mathrm{S}: 71^{\circ} 59^{\prime} \\
57,56^{\circ} \mathrm{O}\end{array}$ & 1980 & 721 & Extendida, Sur & 4 \\
\hline Las Minas & $x$ & $\begin{array}{l}39^{\circ} 55^{\circ} 16,89^{\circ} \mathrm{S}: 73^{\circ} \\
13^{\prime} 54,9^{\circ} \mathrm{O}\end{array}$ & 1978 & 58 & Noreste & 14 \\
\hline Pillo Pillo & $x$ & $\begin{array}{l}39^{\circ} 52^{\prime} 27,41^{\circ} \mathrm{S}: 73^{\circ} \\
06^{\prime} 59,48^{\circ} \mathrm{O}\end{array}$ & 1980 & 44 & Sur & 18 \\
\hline Pumillahue & $\mathrm{x}$ & $\begin{array}{l}39^{\circ} 39^{\prime} 10,52^{\circ} \mathrm{S}: 72^{\circ} \\
46^{\circ} 30^{\circ} \mathrm{O}\end{array}$ & 1982 & 155 & Norte & $4-10$ \\
\hline San Pedro & $\mathrm{x}$ & $\begin{array}{l}39^{\circ} 50^{\prime} 25,83^{\circ} \text { S. } 72^{\circ} \\
49^{\prime} 51.58^{\circ} \mathrm{O}\end{array}$ & 1980 & 45 & Extendida, Norte & 0.2 \\
\hline
\end{tabular}

El tipo de clima y las principales variables climáticas se presentan en el Cuadro $\mathrm{N}^{\circ}$ 3. Todos los rodales presentan temperatura media anual dentro del rango de temperatura media citada en la literatura para la especie, lo mismo ocurre con las temperaturas medias mínimas y máximas. Respecto a la precipitación media anual, también se presentan dentro de los requerimientos de castaño, con una gradiente de norte a sur de alrededor de 1000 $\mathrm{mm}$. Esta diferencia de gradiente junto a los períodos secos, podría marcar una diferencia de crecimiento de las plantaciones dado que castaño tiene altas exigencias hídricas en los meses de verano, en este sentido los rodales de Alupenhue, Lanalhue y Santa Luisa se podrían ver afectados en su desarrollo.

\section{Cuadro $\mathrm{N}^{\circ} 3$ \\ VARIABLES CLIMATICAS DE LOS RODALES DE CASTAÑO ANALIZADOS}

\begin{tabular}{|c|c|c|c|c|c|c|c|}
\hline Rodal & Tipo de Clima & $\begin{array}{l}T \text { modia } \\
\text { anual }\end{array}$ & $\begin{array}{l}T \text { max. } \\
\text { media }\end{array}$ & $\begin{array}{l}T \text { min. } \\
\text { media }\end{array}$ & $\begin{array}{l}\text { Meses secos ylo no } \\
\text { númedos }\end{array}$ & $\begin{array}{l}\text { Pp media } \\
\text { anual }\end{array}$ & $\begin{array}{l}\text { Periodo lare } \\
\text { de helades }\end{array}$ \\
\hline Alupenhue & $\begin{array}{l}\text { Templado mesotermal } \\
\text { inferior estenotermuco } \\
\text { medinerraneo } \\
\text { sublumedo }\end{array}$ & $123^{\circ} \mathrm{C}$ & $26,8^{\circ} \mathrm{C}$ & $4.2^{\circ} \mathrm{C}$ & $\begin{array}{c}\text { periodo seco de } 5 \\
\text { meses }\end{array}$ & $1315 \mathrm{~mm}$ & $\begin{array}{c}11 \text { heladas por } \\
\text { anto }\end{array}$ \\
\hline Lanalinue & $\begin{array}{l}\text { Templado mesotemal } \\
\text { inferior estenotermico } \\
\text { mediterraneo } \\
\text { suohumedo }\end{array}$ & $12.3^{\circ} \mathrm{C}$ & $22.8^{\circ} \mathrm{C}$ & $5.3^{\circ} \mathrm{C}$ & $\begin{array}{c}\text { periodo seco de } 5 \\
\text { meses }\end{array}$ & $1300 \mathrm{~mm}$ & $\begin{array}{c}4 \text { heladas por } \\
\text { affo }\end{array}$ \\
\hline Santa Luisa & $\begin{array}{l}\text { Templado intratermal } \\
\text { estenotermico. } \\
\text { Mediterritneo } \\
\text { subhumedo }\end{array}$ & $10,9^{\circ} \mathrm{C}$ & $22.7^{\circ} \mathrm{C}$ & $4 . \theta^{\circ} \mathrm{C}$ & $\begin{array}{c}\text { periodo soco de } 3 \\
\text { meses }\end{array}$ & $2058 \mathrm{~mm}$ & $\begin{array}{c}8 \text { heladas por } \\
\text { año }\end{array}$ \\
\hline Las Minas & $\begin{array}{l}\text { Marino Fresco. Tipo } \\
\text { Agrocdima Castro }\end{array}$ & $10.49^{\circ} \mathrm{C}$ & ${ }^{19.4^{4} \mathrm{C}}$ & $3.2^{\circ} \mathrm{C}$ & Sin estación seca & $1942 \mathrm{~mm}$ & 5 meses \\
\hline Pillo Plilo & $\begin{array}{l}\text { Marino Fresco: Tipo } \\
\text { Agrodima Loncoche }\end{array}$ & $12.5^{\circ} \mathrm{C}$ & $27.1{ }^{\circ} \mathrm{C}$ & $3.3^{\circ} \mathrm{C}$ & $\begin{array}{c}\text { Enero y Febrero no } \\
\text { humedos }\end{array}$ & $2139 \mathrm{~mm}$ & 3 meses \\
\hline Pumillahue & $\begin{array}{l}\text { Marino Fresco; Tipo } \\
\text { Agroclime Loncoche }\end{array}$ & $12.5^{\circ} \mathrm{C}$ & $27.1^{\circ} \mathrm{C}$ & $3,3^{\circ} \mathrm{C}$ & $\begin{array}{c}\text { Enero y Fabrero no } \\
\text { númedos }\end{array}$ & $2.139 \mathrm{~mm}$ & 3 meses \\
\hline San Pedro & $\begin{array}{l}\text { Marino Fresco, Tipo } \\
\text { agroclima } \\
\text { Loncoche }\end{array}$ & $12,5^{\circ} \mathrm{C}$ & $27,1^{\circ} \mathrm{C}$ & $3.3^{\circ} \mathrm{C}$ & $\begin{array}{c}\text { Enero y Febrero } \\
\text { no humedos }\end{array}$ & $2.139 \mathrm{~mm}$ & 3 meses \\
\hline
\end{tabular}


En relación a las variables edáficas, en el Cuadro $\mathrm{N}^{\circ} 4$ se observa que todos los rodales se encuentran en condiciones edáficas similares, consideradas buenas y dentro de los parámetros que señala la literatura para la especie; suelos profundos, livianos y bien drenados; profundidad entre 50 a más de $1 \mathrm{~m}$, densidad aparente entre 0.42-0.8 g/cm3, y texturas francas, con presencia de horizonte orgánico y litera. Se escapa de estos parámetros el rodal San Pedro el que se localiza en un sitio de condiciones edáficas menos favorables; suelo poco profundo, textura pesada, denso y con poca capacidad de drenaje.

\section{Cuadro 4 \\ VARIABLES EDAFICAS FISICAS DE LOS RODALES DE CASTAÑO ANALIZADOS}

\begin{tabular}{|c|c|c|c|c|c|c|c|}
\hline Rodal & $\begin{array}{l}\text { Prolundidad } \\
\text { der sueio } \mid \mathrm{m} \text { ) }\end{array}$ & Texture & Estructura & $\begin{array}{l}\text { Donsudad } \\
\text { aparente } \\
\text { (plcm') }\end{array}$ & Pedregosidad & $\begin{array}{l}\text { Harzonte } \\
\text { orgáruco } \\
(\mathrm{mm})\end{array}$ & $\begin{array}{l}\text { Litara } \\
\text { (cm) }\end{array}$ \\
\hline Alupentue & $>1$ & $\begin{array}{l}\text { Franco- } \\
\text { Imosal }\end{array}$ & $\begin{array}{l}\text { Grumosa- } \\
\text { subochédrica }\end{array}$ & 08 & $0-25$ & 20 & 6 \\
\hline Lavahue & 08 & $\begin{array}{l}\text { Franco- } \\
\text { arcillose }\end{array}$ & subpohednca & 0.74 & 0.25 & 40 & 8 \\
\hline Senta Luisa & $>1$ & $\begin{array}{l}\text { Franco- } \\
\text { amosa }\end{array}$ & $\begin{array}{l}\text { Gumosa- } \\
\text { subooledrice }\end{array}$ & 0.54 & $0-25$ & 20 & 6 \\
\hline Las Mnas & 08 & $\begin{array}{l}\text { Franco- } \\
\text { Irmosa }\end{array}$ & $\begin{array}{l}\text { Grumosa- } \\
\text { suboolidonca }\end{array}$ & 0.69 & $0-25$ & 20 & 5 \\
\hline Pillo Pilo & $>1$ & $\begin{array}{l}\text { Franca- } \\
\text { imosa }\end{array}$ & $\begin{array}{l}\text { Grumosa. } \\
\text { subpollearica }\end{array}$ & 0.59 & 0.25 & 15 & 5 \\
\hline Punillahue & $>1$ & $\begin{array}{l}\text { Franco- } \\
\text { Imosa }\end{array}$ & $\begin{array}{l}\text { Grumoes- } \\
\text { subpoleance }\end{array}$ & $0 . \overline{42}$ & 0.25 & 30 & 5 \\
\hline San Pedro & $<05$ & $\begin{array}{l}\text { Limo- } \\
\text { arcillosa }\end{array}$ & $\begin{array}{l}\text { MasNa } \\
\text { (suopoliedrica) }\end{array}$ & 1.16 & $50-75$ & 10 & 5 \\
\hline
\end{tabular}

Respecto al análisis químico se observa que todos los suelos de los rodales analizados presentan $\mathrm{pH}$ moderado, buena presencia de materia orgánica y que no son deficitarios en macrolelementos, a excepción del Fósforo. Solo Alupenhue no presenta déficit de Fósforo, esto se explica debido a que el suelo de este rodal es de origen volcánico y corresponde a un suelo Tipo Trumao, que se caracteriza por fijar Fósforo, lo que no significa que este elemento esté disponible para las plantas. El rodal San Pedro es el único que podria ser considerado deficitario en macronutrientes, lo que se explicaria por ser el único que presenta una bajo presencia de materia orgánica. Por otra parte en todas las situaciones, los suelos presentan déficit principalmente de Boro. En relación al Calcio el rodal que presenta niveles elevados para la especie es Alupenhue y, en el caso de Aluminio, el rodal que presenta mayor concentración es Las Minas, niveles que pudieran presentar alguna toxicidad y representar un riesgo de mortalidad. 


\section{Cuadro $\mathrm{N}^{\circ} 5$}

\section{ANALISIS QUIMICO DE SUELOS DE LOS RODALES DE CASTAÑO ANALIZADOS}

\begin{tabular}{|c|c|c|c|c|c|c|c|c|c|c|c|c|c|c|}
\hline \multirow[t]{2}{*}{ identeficación } & \multirow{2}{*}{$\begin{array}{l}\mathrm{pH} \\
\text { agua }\end{array}$} & $B$ & $\begin{array}{l}\mathrm{P} \\
\text { Otsen }\end{array}$ & s & Ca & $M g$ & $\mathrm{Na}$ & k & Al & \multirow{2}{*}{$\begin{array}{l}\text { Suma } \\
\text { Basers }\end{array}$} & \multirow{2}{*}{\begin{tabular}{|l}
$\mathrm{Ct}$ \\
$\mathrm{x}$
\end{tabular}} & \multirow{2}{*}{$\begin{array}{l}\text { M ORG } \\
\alpha\end{array}$} & \multirow{2}{*}{$\begin{array}{l}\mathrm{Nt} \\
\mathrm{g} \\
\end{array}$} & \multirow{3}{*}{$\begin{array}{c}\text { CIN } \\
28,7\end{array}$} \\
\hline & & \multicolumn{8}{|c|}{ mglkg } & & & & & \\
\hline Alucoenture & 6.21 & 0.14 & 16.7 & 5 & 1832 & 168 & 12 & 545 & 3 & 12.01 & 8,62 & 14.9 & 0.30 & \\
\hline Lanahue & 5.33 & 047 & 39 & 12 & 1011 & 303 & 24 & 268 & 38 & 8.37 & 6,99 & 121 & 0.32 & 21.8 \\
\hline Santa Lusa & 583 & 004 & 28 & 10 & 266 & 34 & 9 & 109 & 6 & 1.93 & 7.65 & 132 & 033 & 232 \\
\hline Las Minas & 5.04 & 0.41 & 4.9 & 22 & 21 & 18 & 13 & 67 & 84 & 0,48 & 8.38 & 144 & 0.40 & 21.0 \\
\hline Pillo Pillo & 501 & 0.23 & 48 & 17 & 94 & 31 & 10 & 81 & 45 & 0.98 & 8.65 & 149 & 0.46 & 18.8 \\
\hline Pumilatue & 5.65 & 0.06 & 3 & 13 & 359 & 71 & 17 & 52 & 7 & 2.59 & 8.39 & 145 & 0.49 & 17.1 \\
\hline San Pedro & 5.90 & 0.28 & 45 & 2 & 1060 & 222 & 17 & 133 & 9 & 756 & 3.90 & 67 & 096 & 244 \\
\hline
\end{tabular}

Las variables dasométricas, son presentadas en el Cuadro $N^{\circ} 6$ y las variables de de forma y sanidad en el Cuadro $\mathrm{N}^{0} 7$.

\section{Cuadro $\mathrm{N}^{\circ} 6$}

\section{VARIABLES DASOMETRICAS DE LOS RODALES DE CASTAÑO ANALIZADOS}

\begin{tabular}{|l|c|c|c|c|c|c|c|}
\hline Rodal & $\begin{array}{c}\text { Ano } \\
\text { Plantación }\end{array}$ & $\begin{array}{l}\text { Dap } \\
(\mathrm{cm})\end{array}$ & $\begin{array}{l}\text { Incremento } \\
\text { medio anual } \\
\text { DAP }(\mathrm{cm})\end{array}$ & Dominancia & $\begin{array}{l}\text { Altura total } \\
(\mathrm{m})\end{array}$ & $\begin{array}{l}\text { Incremento } \\
\text { medio anual } \\
\text { Altura }(\mathrm{m})\end{array}$ & $\begin{array}{c}\text { Cobertura } \\
\text { Copas } \\
\%\end{array}$ \\
\hline Alupenhue & 1998 & 8,7 & 1,24 & 2,0 & 10,0 & 1,42 & $75-100$ \\
\hline Lanalhue & 1979 & 25,9 & 1.0 & 1,4 & 22,3 & 0,86 & $75-100$ \\
\hline Santa Luisa & 1980 & 16,1 & 0,65 & 1,7 & 15,9 & 0,64 & $75-100$ \\
\hline Las Minas & 1978 & 23,4 & 0,86 & 1,5 & 21,4 & 0,79 & $75-100$ \\
\hline Pillo-Pillo & 1981 & 21,5 & 0,89 & 1,8 & 25,7 & 1,07 & $75-100$ \\
\hline Pumillahue & 1983 & 24,2 & 1,1 & 1,6 & 20,2 & 0,92 & $75-100$ \\
\hline San Pedro & 1980 & 25,8 & 1,03 & 1,4 & 21,0 & 0,84 & $50-75$ \\
\hline
\end{tabular}

Nota: criterios de asignación

Dominancia: 1: Dominante; 2: Codominante; 3: Intermedio; 4: Suprimido

Dado que los rodales presentan distintas edades es dificil hacer comparaciones en términos de crecimiento. Sin embargo, los estudios existentes en el país sobre crecimiento de la especie, muestran incrementos medios anuales al rededor de $1 \mathrm{~cm}$ de diámetro y de 1 $\mathrm{m}$ de altura. En este contexto la mayoria de los rodales analizados cumplen con esta caracteristica, a excepción de Santa Luisa. 


\section{Cuadro $\mathrm{N}^{\circ} 7$}

VARIABLES DE FORMA Y DE SANIDAD DE LOS RODALES DE CASTAÑO ANALIZADOS

\begin{tabular}{|l|c|c|c|c|}
\hline Rodal & Año Plantación & Sanidad & Rectitud & Flecha \\
\hline Alupenhue & 1998 & 1,2 & 2,0 & 0,3 \\
\hline Lanalhue & 1979 & 1,1 & 1,5 & 0,5 \\
\hline Santa Luisa & 1980 & 1,1 & 1,7 & 0,3 \\
\hline Las Minas & 1978 & 1,0 & 2,1 & 0,4 \\
\hline Pillo-Pillo & 1981 & 1,0 & 2,2 & 0,5 \\
\hline Pumillahue & 1983 & 1,0 & 1,9 & 0,2 \\
\hline San Pedro & 1980 & 1,1 & 2,0 & 0.2 \\
\hline
\end{tabular}

Sanidad: 1: Sano; 2: Regular; 3: Malo

Rectitud: 1: Bueno; 2: Regular; 3: Malo

Flecha: 1: dos o más flechas: 0 : sin doble flecha

En relación a las caracteristicas de forma, los rodales analizados presentan forma regular en rectitud y un porcentaje importante de presencia de flechas. Esto se debería a que en general el manejo de estos rodales ha sido deficitario o nulo.

En cuanto a sanidad, se observa que todos los rodales presentan buenas condiciones (valores muy cercanos a 1, que representa el criterio de sano). Esto indica que en el área de estudio, que corresponde a las zonas definidas como aptas para la especie en Chile (Benedetti y Subiri, 200), castaño no es afectado en niveles importantes por agentes patógenos como en otras partes del mundo, en que éste se caracteriza por tener serios problemas sanitarios, principalmente por ataque de hongos del género Phytophtora, presente en Chile, y Endothia, no presente en Chile a la fecha.

Finalmente, en relación a la vegetación acompañante en los rodales analizados, como se observa en el Cuadro $\mathrm{N}^{\circ} 8$, a excepción del rodal de Alupenhue, ésta es la misma en todos los rodales. Esto se explica debido a que las especies nativas presentes corresponden a parte de la vegetación nativa caracteristica de los distintos lugares en que se ubican estas plantaciones. En cuanto a la presencia de rosa mosqueta y zarzamora, son especies invasoras presentes en una amplia superficie en Chile. 


\section{Cuadro $N^{\circ} 8$ \\ VEGETACION ASOCIADA A LOS RODALES DE CASTAÑO ANALIZADOS}

\begin{tabular}{|l|c|l|}
\hline Rodal & Año Plantación & \multicolumn{1}{|c|}{ Vegetación acompañante } \\
\hline Alupenhue & 1998 & Malezas, gramineas \\
\hline & 1979 & $\begin{array}{l}\text { Arbustiva nativa Radal avellanillo, maqui } \\
\text { Arborea nativa: renovales de lingue y avellano Regeneracion } \\
\text { castaño, rosa mosqueta, zarzamora }\end{array}$ \\
\hline Santa Luısa & 1980 & $\begin{array}{l}\text { Arbustiva nativa Radal avellanillo, maqui } \\
\text { Arborea natıva. renovales de lingue avellano Regeneracion } \\
\text { castaño, rosa mosqueta, zarzamora }\end{array}$ \\
\hline Las Mınas & 1978 & $\begin{array}{l}\text { Arbustiva nativa Radal avellanillo, maqui } \\
\text { Arborea nativa renovales de lingue avellano Regeneracion } \\
\text { castaño, rosa mosqueta, zarzamora }\end{array}$ \\
\hline Pillo-Pilo & 1981 & $\begin{array}{l}\text { Arbustiva nativa:Radal avellanillo, maqui } \\
\text { Arborea nativa. renovales de lingue avellano Regeneracion } \\
\text { castaño, rosa mosqueta, zarzamora }\end{array}$ \\
\hline Pumillahue & 1983 & $\begin{array}{l}\text { Arbustiva nativa Radal avellanillo, maqui } \\
\text { Arborea nativa: renovales de lingue avellano Regeneracion } \\
\text { castaño, rosa mosqueta, zarzamora }\end{array}$ \\
\hline & 1980 & $\begin{array}{l}\text { Arbustiva nativa. Radal avellanillo, maqui } \\
\text { Arborea nativa renovales de lingue avellano Regeneracion } \\
\text { castaño, rosa mosqueta, zarzamora }\end{array}$ \\
\hline
\end{tabular}

Aún cuando es necesario hacer un estudio más acabado sobre la interrelación especifica y la dinámica vegetacional, la presencia de estas especies en plantaciones de castaño podría indicar que éstas se asocian bien a castaño, pudiendo convivir en un mismo espacio. Lo anterior junto a la presencia de regeneración de castaño, representan elementos de análisis interesantes en la definición de propuestas de manejo silvicultural, para tipos de formaciones de monte alto o monte bajo, en esquemas mixtos que puedan dar mayor sostenibilidad económica y ambiental.

\section{CONCLUSIONES}

En general, las variables ambientales en que se localizan los rodales estudiados, están dentro de los rangos de los requerimientos ecológicos que cita la literatura nacional e internacional de para castaño.

Lo anterior da cuenta de la plasticidad de la especie, en cuanto es capaz de crecer en una amplia variedad de sitios.

El buen estado sanitario observado en estos rodales, considerando la amplitud geográfica en que estos se distribuyen, ratifica la inexistencia a la fecha de agentes de daño importantes para castaño en Chile.

El buen desarrollo en diámetro y altura de los castaños analizados y los resultados en relación a las variables de forma, junto al escaso manejo silvicultural del que han sido 
objeto, indicaría que la aplicación de manejo adecuado orientado a la producción de madera de alto valor es posible.

Lo observado en relación a la vegetación acompañante en los rodales analizados y la presencia de la regeneración de la especie, permitiria orientar propuestas de esquemas de manejo forestal.

Como conclusión final, los antecedentes generados en este estudio, confirman el interesante potencial de castaño para la producción forestal en Chile.

\section{REFERENCIAS}

Amorini, E.; Bruschini, S; Fioravanti, M; Macchioni, N; Manetti, M.C.; Thibaut, B; Uzielli, L., 1997. Studi Sulle Cause di Insorgenza della Cippllatura del Legno di Castagno. Convegno Nazionale sul Castagno. Cison di Valmarino. Treviso. Pp $269-290$.

Bagnaresi, U. 1986. II Castagno da Frutto. II Divulgatote $N^{\circ}$ 28. Serie Regione Emilia Romagna. 52 p.

Benedetti, S.; Subiri, M., 2000. El Castaño: Una Opción de Producción Forestal. INFOR. Santiago, Chile. 93 p.

Benedetti S., Saavedra, J., 2003. Estrategia de Transferencia Tecnológica para la Creación de Plantaciones de Castaño: Sistematización de una Experiencia Exitosa. INFOR. Santiago, Chile. 22 p.

Berrocal, M., Gallardo, J. y Cardeñoso, J., 1998. El Castaño. Productor de Fruto y Madera. Creador de Paisaje y Protector. Ediciones Mundi - Prensa. Madrid. 228 p.

Bourgeois, C., 1992. Le Chataigneir. Un Arbre, Un Bois. Institut pour le Developpement Forestier. Paris, Francia. 367 p.

Cabrera, J. y Meneses, M., 1998. Diversificación Forestal: Costos, Usos y Productos Forestales. Informe Módulo de Comercialización. Proyecto INFOR-Fondef D96I1055. Documento Interno. INFOR. Santiago, Chile. 20 p.

Juacida, R., Diaz-Vaz, J. E., Poblete, H., Rodríguez, S. y Cuevas, H., 1999. Caracterización Tecnológica de Castaño, Encino y Ciprés y Opciones de Uso. UACH. Valdivia, 12 p.

Loewe, V., Neuenschwander, A. y Alvear, C., 1994. El Castaño en Chile: Un Cultivo Fruto Forestal Promisorio. Documento Técnico $\mathrm{N}^{\circ} 85$. INFOR. $61 \mathrm{p}$.

Macchioni, N. y Pividori, M., 1996. Qualitá del Legno del Ceduo di Castagno: Gestione dei Popolamenti. Silvae Pedemontis. 2(1): $23-30$.

Medel, F. 1986. Requerimientos Climáticos y Edáficos para las Especies Frutales en el Sur de Chile. Agrosur. 14 (1): $48-56$.

Parra, P. y González, M., 1998. La Chicharra. Informativo Sanitario Forestal N 1. Grupo 1 Insectos. INFOR. Santiago, Chile. 
Zanuttini, P. y Cielo, P., 1996. Caratteristiche Tecnologiche ed Impieghi del Legno de Castagno. Silvae Pedemontis. Semestrale della Associazione Forestale del Piemonte. 2 (1). 31 p. 
